\title{
Reducing unnecessary fixation of midshaft clavicle fractures
}

Introduction:

- Displaced midshaft clavicle fractures have a nonunion rate of $10-20 \%$. Those who unite with conservative treatment have similar outcomes to those who undergo operative treatment, therefore protocols to identify potential non-unions are important to avoid unnecessary surgery.

Aim:

- To report of on one such protocol

Figure 1

Middle third clavicle fracture outpatient management

\section{Is there}

An open clavicle fracture A floating shoulder Multiple rib fractures Compromised skin A pathological fracture Neurovascular injury

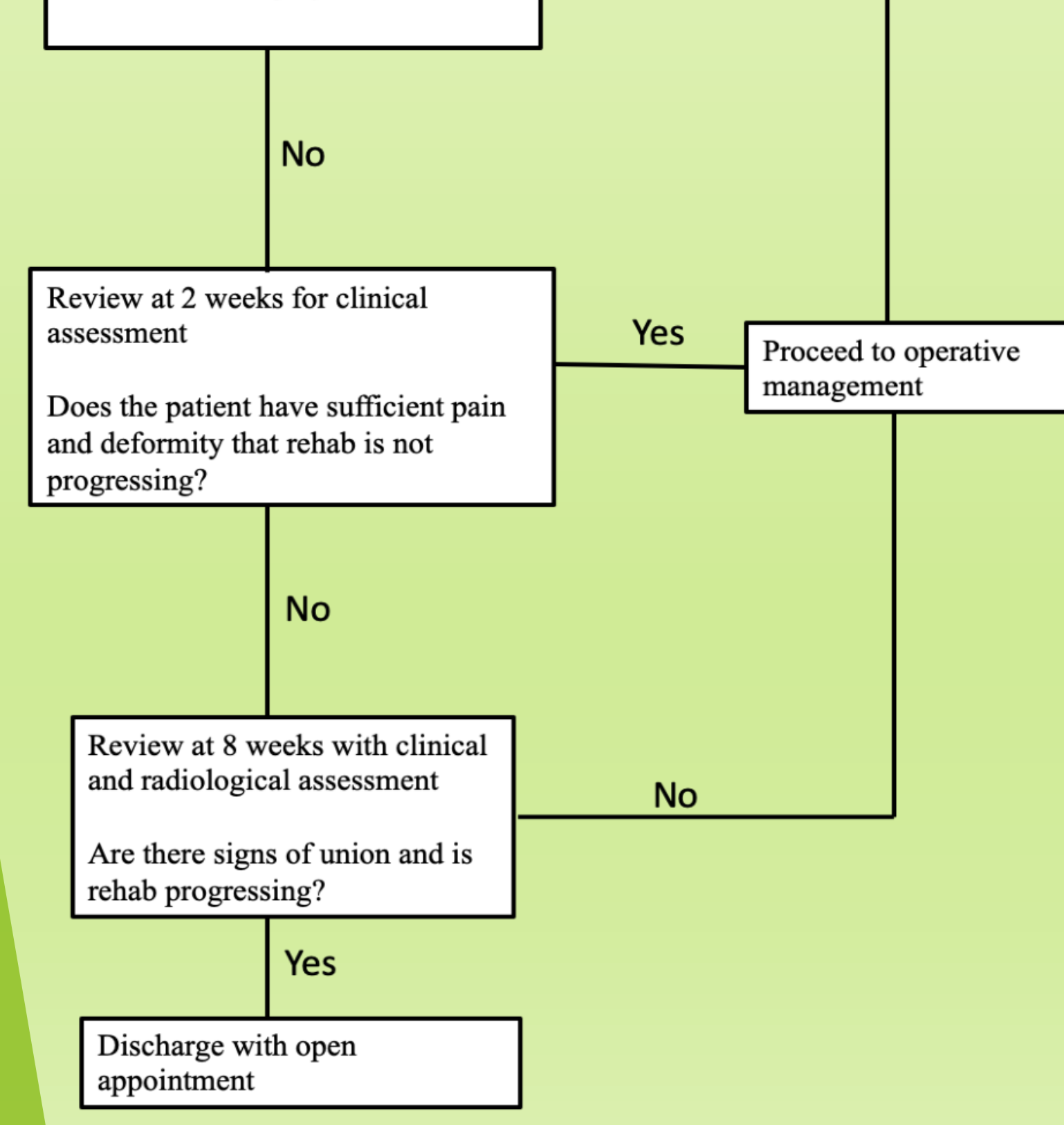

Authors: Murray NJ, Johnson T, Packham IN, Crowther MAA, Chesser TJS North Bristol NHS Trust, Bristol, UK

No Disclosures

Patients and Methods:

A protocol was introduced, where all isolated closed displaced midshaft clavicle fractures were initially managed non-operatively in a sling. At 2 weeks the patients were reviewed and those who were struggling with their symptoms were offered surgery, with the remainder mobilised as comfortable. All cases treated at one centre over a threeyear period, with a minimum follow up of one-year underwent case note review. See figure 1.

\section{Results:}

Between 2015 and 2017613 clavicle fractures were managed through clinic as demonstrated in table 1. 41 middle third clavicle fracture patients underwent early fixation. 8 were excluded as they were managed out with the protocol eg open fracture, tented skin. The number of days to operation

\section{Table}

\begin{tabular}{ll}
\hline Total clavicle fractures & 613 \\
\hline Medial & $11(2 \%)$ \\
Middle & $347(56 \%)$ \\
\hline Distal & $255(42 \%)$ \\
\hline Middle third clavicle fractures & \\
\hline Male & $262(75 \%)$ \\
\hline Female & $85(25 \%)$ \\
\hline Mean Age & $41 y($ range $16-97 y)$ \\
\hline Displaced & $225(65 \%)$
\end{tabular}

Displaced

$41 \mathrm{y}$ (range 16-97y)

was 4-57 with a median of 15 days. 11 patients required late fixation for symptomatic delayed, non or mal union. 4 were excluded as they were treated outside the protocol eg from another hospital. Late surgery occurred at a mean of 378 days (range 193-494 days), 6 for symptomatic non-unions and 1 was a symptomatic malunion. The early operative rate was $10 \%$ (33 of 335 patients), and non-union or symptomatic malunion rate was $2 \%$. This led to a total operative rate of $12 \%$. See figure 2 .

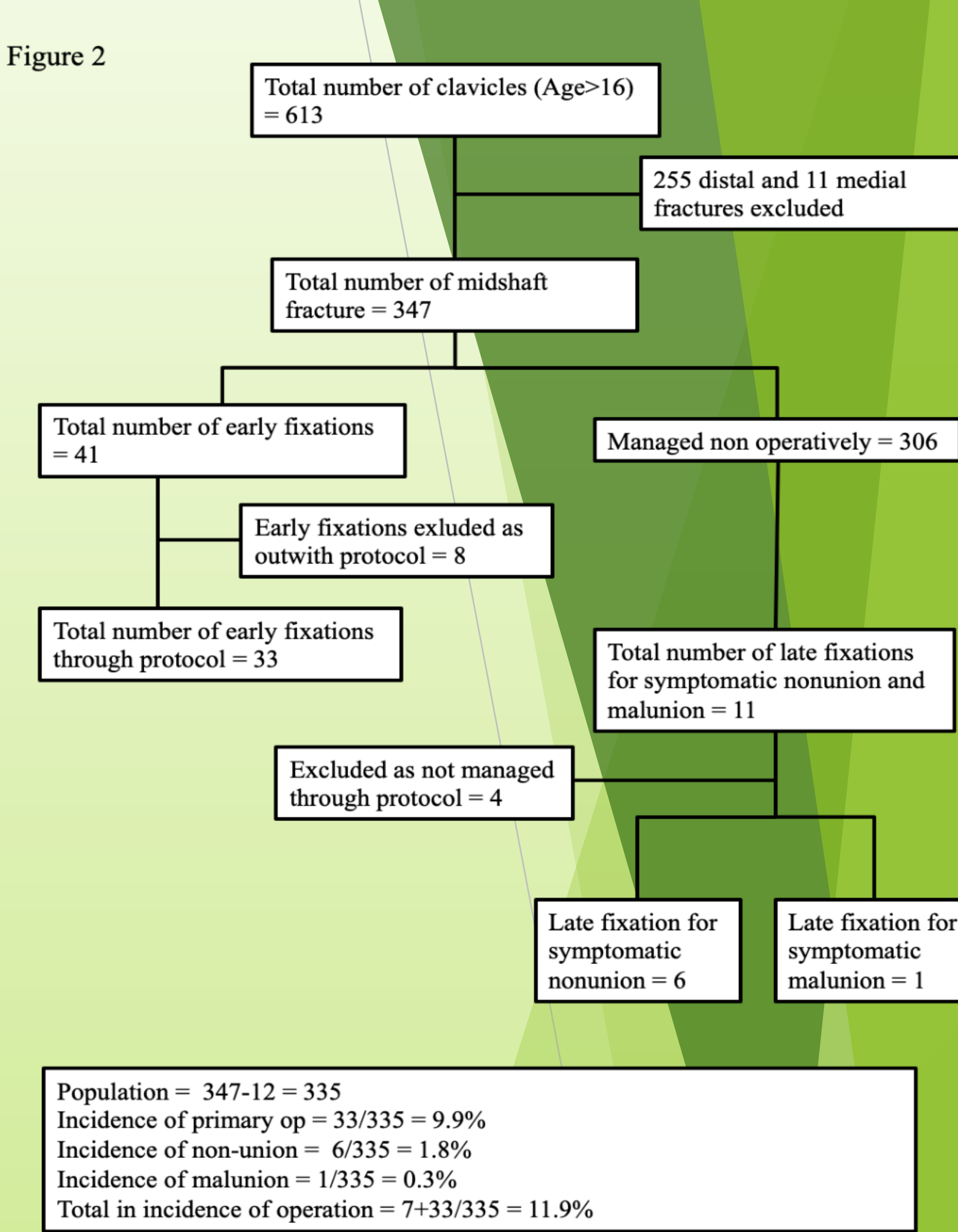

Conclusions:

A protocol for managing middle third clavicle fractures has demonstrated an effective means of managing these injuries. It is cost effective reducing the number of patients requiring fixation with a fixation rate of $12 \%$ whilst reducing the rate of symptomatic non and malunion (2\%). The management pathway is simple, and could be introduced into any orthopaedic outpatient department with ease.

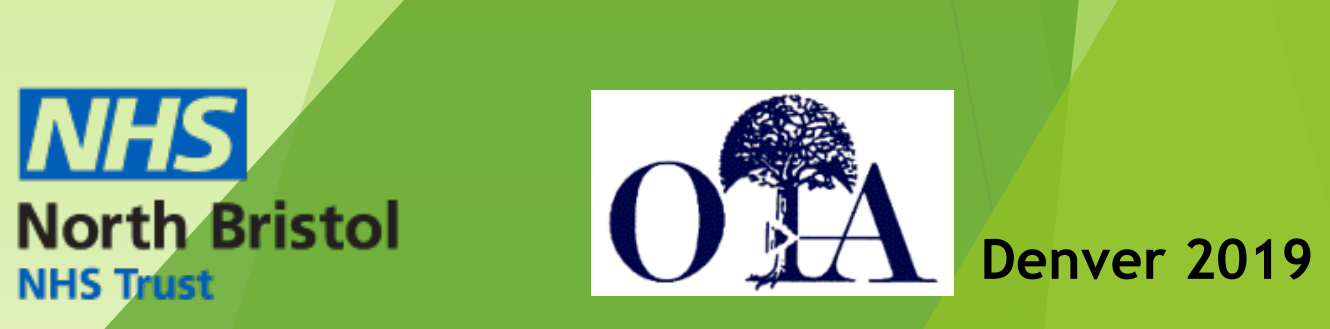

